\title{
Genesis
}

Manuscrits - Recherche - Invention

$51 \mid 2020$

Intertextualité - Exogenèse

\section{L'exposition « Pascal Quignard, fragments d'une écriture » à la Galerie des donateurs de la BnF}

\section{Irène Fenoglio}

\section{(2) OpenEdition}

1 Journals

\section{Édition électronique}

URL : https://journals.openedition.org/genesis/5592

DOI : 10.4000/genesis.5592

ISSN : 2268-1590

\section{Éditeur :}

Presses universitaires de Paris Sorbonne (PUPS), Société internationale de génétique artistique littéraire et scientifique (SIGALES)

Édition imprimée

Date de publication : 15 décembre 2020

Pagination : 233-234

ISBN : 979-10-231-0704-3

ISSN : 1167-5101

\section{Référence électronique}

Irène Fenoglio, «L'exposition «Pascal Quignard, fragments d'une écriture » à la Galerie des donateurs de la BnF », Genesis [En ligne], 51 | 2020, mis en ligne le 10 janvier 2021, consulté le 07 février 2022. URL : http://journals.openedition.org/genesis/5592 ; DOI : https://doi.org/10.4000/genesis.5592

Ce document a été généré automatiquement le 7 février 2022.

Tous droits réservés 


\title{
L'exposition « Pascal Quignard, fragments d'une écriture » à la Galerie des donateurs de la BnF
}

\author{
Irène Fenoglio
}

1 En 2018, Pascal Quignard a fait don de ses archives à la Bibliothèque nationale de France. Laurence Engel, présidente de cette vénérable institution, s'attarde sur le geste singulier de l'auteur, tout en le replaçant dans « une histoire, scandée par ceux qui ont fait de la BnF leur Panthéon littéraire, leur Panthéon volontaire. De Bossuet à Roland Barthes, de Victor Hugo à Marcel Proust ou encore de Marie Curie à Nathalie Sarraute ${ }^{1} . »$ Si une panthéonisation ne convient guère à la personnalité de Pascal Quignard ( "J'ai vu des hommes qui prétendaient s'être fait un nom. Mais qu'est-ce qu'un nom dans le réel? Qu'est-ce que le petit mot ego au début de la phrase? Tellement moins qu'un oiseau qui chante ${ }^{2} »$ ), il est néanmoins l'un des auteurs qui a le plus déployé d'écrits sur la lecture, l'écriture et le livre; et qui a le plus intégré, protégé, au sein de ses livres, lecteurs, écrivains, penseurs premiers, anciens, classiques. L'acte d'écrire et de lire, ainsi que leurs longues traditions constituent le sol, le socle, et le cœur constant de ses écrits. En témoigne encore son dernier livre, L'homme aux trois lettres ${ }^{3}$. En regard de l'ampleur de cette œuvre - qui accueille en son sein l'histoire de la langue française ainsi que la très longue histoire sans origine de la littérature -, en regard de sa profondeur et de son aura culturelle, seule la Bibliothèque nationale était à sa mesure.

2 Pour rendre hommage à ce don, fait du vivant de l'auteur et alors qu'il est toujours en pleine écriture, la BnF a organisé, du 30 septembre au 29 novembre 2020, à la Galerie des donateurs, une exposition de documents personnels, dessins, objets et manuscrits de Pascal Quignard. Comme le souligne Olivier Wagner, commissaire de l'exposition, celle-ci «ne vise pas à l'exhaustivité [...], elle n'en fournit qu'un instantané incomplet $t^{4}$ ». L'exposition, intitulée "Pascal Quignard, fragments d'une écriture», se concentre autour des manuscrits de Boutès. 
3 Il faut savoir que parmi l'ensemble des archives qui ont fait l'objet du don, ainsi que dans l'exposition, les manuscrits proprement textuels sont peu nombreux en comparaison de l'œuvre immense de l'auteur - une centaine de livres en cinq décennies -, car l'auteur ne les conserve pas, comme lui-même l'a exprimé à plusieurs reprises, notamment dans le film de Jacques Malaterre À mi-mots ${ }^{5}$.

4 Les manuscrits qui ont été conservés ont presque tous été étudiés à l'ITEM, ceux de Boutès précisément, qui ont donné lieu à un ouvrage de génétique textuelle, Sur le désir de se jeter à l'eau, écrit à deux voix : celle de l'auteur et celle de la généticienne ${ }^{6}$.

5 Le numéro 27 de Genesis, en 2006, inaugurait l'étude d'un manuscrit de Quignard, celui du conte Fête des Chants du Marais. Il s'agissait alors de montrer - entre autres - qu'un auteur contemporain utilisant l'ordinateur et son «traitement de texte» constituait bien un dossier de genèse : un ensemble de notes, feuillets, documents mêlant écriture manuscrite et tapuscrite s'acheminant vers un texte final publiable sinon publié, et élaborait un manuscrit, c'est-à-dire cet ensemble hybride de matériau verbal textuel, de signes graphiques divers, de semiosis complexe pouvant comprendre des dessins, et constituant l'espace privilégié où les traces de l'élaboration scripturale sont observables. En l'occurrence, le texte final de ce conte écrit en 2006 ne trouva place et forme finales qu'en 2009, dans l'ensemble que constitue Dernier Royaume ${ }^{7}$. Il fut repris ensuite sous la forme d'un livret de concert-fiction, «Les Enfants du Marais », sur une musique de Suzanne Giraud ${ }^{8}$. L'article de Genesis relatant la genèse non définitive de ce texte faisait émerger la notion de "genèse in vivo » définie de la façon suivante : « un grand écrivain de notre tout jeune $\mathrm{xxI}^{\mathrm{e}}$ siècle, promis à la postérité de l'écriture universelle, nous offrait - dans le même temps qu'il l'écrit et qu'il tient son texte sous le coude - la liberté absolue d'en ouvrir le manuscrit toujours vivant ${ }^{9}$.»

Entre temps, Pascal Quignard avait accepté de conserver tous les éléments (manuscrits comme documents) relatifs à un texte qu'il était en train d'écrire, expliquant qu'il accumulerait un document ou feuillet l'un sur l'autre au fur et à mesure de leur « sortie "; le plus ancien se trouvait ainsi au-dessous de la pile, et le plus récent tout en haut. Une lettre de l'auteur - qui est exposée à la BnF - témoigne de cette démarche volontaire de sa part rompant avec son habitude de ne pas garder ses manuscrits le texte une fois achevé et publié. Car c'est bien ce que fait l'auteur, il ne conserve aucun manuscrit hormis, parfois, quelques feuillets illustrés de sa main. L'exposition de la BnF offre aux regards, par exemple, les magnifiques feuillets sauvés du manuscrit de Tous les matins du monde.

7 C'est ainsi grâce à la collaboration de l'auteur et d'une chercheuse en génétique qu'a pu être constitué et totalement conservé le manuscrit d'un livre important: conférence sur la musique au départ, le texte est finalement publié en 2008, chez Galilée, sous le titre définitif de Boutès. L'auteur le précise dans le catalogue d'exposition Sur le geste de l'abandon $^{10}$ : il s'agira là du seul manuscrit conservé dans sa totalité.

8 C'est un ensemble rare et inestimable puisqu'il est le seul dossier de genèse complet d'un texte de Pascal Quignard et que ce dossier est entièrement publié dans Sur le désir de se jeter à l'eau, dialogue fructueux entre l'auteur et la généticienne.

9 Avant l'événement actuel proposé par la BnF, deux expositions avaient été organisées autour de ce précieux ensemble: la première à Guéret lors des $5^{\text {es }}$ Rencontres de Chaminadour consacrées à Pascal Quignard en septembre 2010 ; la seconde à Cerisy-la- 
Salle lors du colloque "Pascal Quignard. Translations et métamorphoses » en juillet $2014^{11}$. Ces deux expositions portaient le même titre : "La part du retiré ».

Aujourd'hui, les manuscrits de Boutès et tout ce qui se rapporte à l'écriture de ce texte (correspondance, illustrations, sculpture, publications) constituent le cœur de l'exposition de la Bibliothèque nationale.

\section{NOTES}

1. Laurence Engel, «Faites entrer l'infini », dans Pascal Quignard, Sur le geste de l'abandon, Paris, éd. Hermann, p. 3.

2. Pascal Quignard, L'homme aux trois lettres, Paris, Grasset, 2020, p.68. L'ouvrage constitue le tome XI de Dernier Royaume.

3. Ibid.

4. Olivier Wagner, «Fragments d'une écriture », dans Pascal Quignard, Sur le geste de l'abandon, op. cit., p. 8. Voir aussi « Pascal Quignard, fragments d'une écriture », Chroniques, $n^{\circ} 88-89$, Paris, BnF, 2020, p. 14-15.

5. Jacques Malaterre, À mi-mots. Pascal Quignard, production Arte France-MK2 TV, 2001.

6. Pascal Quignard, Irène Fenoglio, Sur le désir de se jeter à l'eau, Paris, PSN, 2011.

7. Le texte est repris dans La Barque silencieuse, t. vi de Dernier Royaume, et sous le titre Les fêtes des chants du marais, Paris, Grasset, 2009, p. 136-141.

8. Création à Paris le 19 octobre 2019, par l'orchestre philarmonique de Radio France et la Maîtrise de Radio France, sous la direction de Suzanne Giraud.

9. Irène Fenoglio, « Fête des Chants du Marais, un conte inédit de Pascal Quignard. Genèse in vivo et traitement de texte", Genesis, $n^{\circ} 27,2006$, p. 73, en ligne sur persee.fr. Ce même numéro de Genesis publiait un entretien avec l'auteur intitulé « ...ce vivre-écrire que je suis... », en ligne sur persee.fr.

10. Pascal Quignard, Sur le geste de l'abandon, op. cit., p. 92.

11. Colloque «Pascal Quignard. Translations et métamorphoses", organisé par M. Calle-Gruber, J. Degenève et I. Fenoglio, Cerisy-la-Salle, 9-16 juillet 2014, en ligne sur ccic-cerisy.asso.fr. Voir la publication des textes du colloque avec CD audio, sous le même titre aux éditions Hermann, 2015.

\section{AUTEUR}

\section{IRÈNE FENOGLIO}

Directrice de recherche émérite, IRÈNE FENOGLIO dirige l'équipe « Linguistique » de l'ITEM.

Spécialiste de manuscrits de linguistes (en particulier ceux d'Émile Benveniste dont elle a édité les Dernières Leçons, Seuil, 2012), elle s'est aussi intéressée aux manuscrits d'auteurs contemporains (dont Louis Althusser, Erri de Luca, Pascal Quignard). Elle est l'auteur de très 
nombreux articles et ouvrages dont L'Écriture et le Souci de la langue (Academia-Bruylant, 2007), Une Auto-graphie du tragique (Academia-Bruylant, 2007), Sur le désir de se jeter à l'eau avec Pascal Quignard (PSN, 2011), Le Geste linguistique (Genesis, n 35), Autour d'Émile Benveniste (Seuil, 2016), Émile Benveniste, 50 ans après les PLG (Rue d'Ulm, 2019). 